\title{
Design study of a Split-Coaxial RFQ for IsoDAR
}

\author{
Jungbae Bahng ${ }^{1}$ \\ Korea University Sejong campus \\ 2511 Sejong-ro, Sejong 30019, South Korea \\ E-mail: bahngjb@korea.ac.kr \\ Daniel Winklehner \\ Massachusetts Institute of Technology \\ 77 Massachusetts Ave., Cambridege, MA 02139, USA \\ E-mail: winklehnemit.edu

\section{Janet Conrad} \\ Massachusetts Institute of Technology \\ 77 Massachusetts Ave., Cambridege, MA 02139, USA \\ E-mail: conrademit.edu
}

\begin{abstract}
The Isotope Decay-At-Rest experiment (IsoDAR) is a proposed experiment to search for sterile neutrinos by measuring neutrino oscillations. The electron-antineutrino generation requires a high intensity primary proton beam impinging on a beryllium target surrounded by lithium. In IsoDAR, $\mathrm{H} 2+$ ions are generated and accelerated to avoid space charge effects in the low energy region, which will be stripped into protons after extraction from a cyclotron. As part of the IsoDAR injection system, an RFQ buncher with $32.8 \mathrm{MHz}$ of operation frequency provides $70 \mathrm{keV}$ acceleration and strong bunching of the $\mathrm{H} 2+$ beam. The RFQ will be installed halfway inside the iron yoke of the cyclotron to be very close to the median plane. Because the beam starts diverging after the RFQ in both transverse and longitudinal direction, a re-buncher is employed in the end transition cell to re-focus the beam longitudinally. In this paper, we describe in detail the beam dynamics study and RF analysis of the IsoDAR RFQ for direct injection into a compact cyclotron.
\end{abstract}

ICHEP2018, International conference on High Energy Physics

4-11 July 2018

Seoul, South Korea

\section{${ }^{1}$ Speaker}




\section{Introduction}

The RFQ-Direct Injection Project (RFQ-DIP) aims to build a compact injection system for the IsoDAR experiment, which is a proposed search for sterile neutrinos by measuring neutrino oscillations. The primary neutrinos are produced by a proton beam striking a Be target. The IsoDAR driver is composed of an ion source, an RFQ accelerator and a cyclotron [1]. The RFQ has been developed as part of the IsoDAR injection system to increase capture efficiency in the cyclotron by efficient longitudinal bunching. An added benefit is the reduction of extraction voltage from the ion source. Fig. 1 shows a conceptual layout of the IsoDAR injection system. Beam is injected directly into the cyclotron via a spiral inflector in the central region. In order for the RFQ-DIP to be successful, an RFQ has many design goals such as a small cavity size to install the RFQ inside the cyclotron yoke, a small longitudinal emittance for good acceptance in the cyclotron, re-bunching for minimizing the bunch length increase between RFQ and cyclotron, and short length of cavity for easy installation and alignment. In the following sections, we describe the beam dynamics study and cavity design of this novel RFQ for the RFQ-DIP.

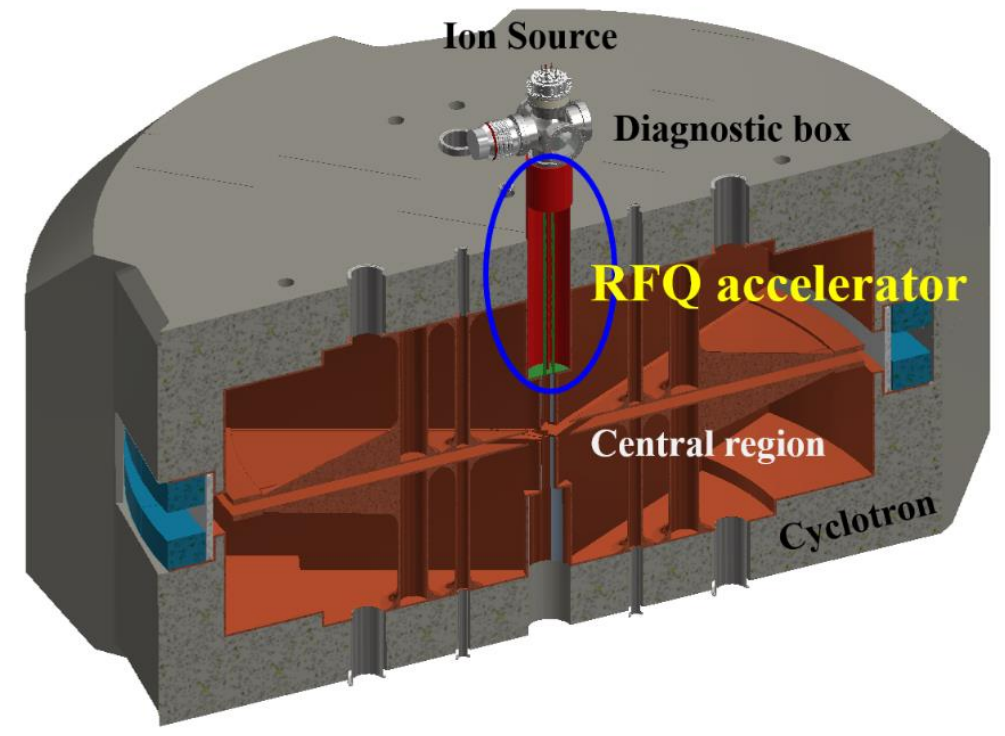

Figure 1 : Conceptual layout of the IsoDAR injection system

\section{RFQ beam dynamics}

To increase the acceptance, the particles coming from the ion source need to be bunched before being injected into an RF accelerator like a cyclotron. An RFQ is the best device to capture beam with high efficiency by bunching adiabatically in the low energy section. An RFQ can provide over $90 \%$ of bunching efficiency and also accelerate the particles. The design parameters of the beam dynamics are listed in Table. 1. The IsoDAR RFQ has been designed to accelerate $\mathrm{H}_{2}{ }^{+}$ions from the injection energy of $15 \mathrm{keV}$ up to $70 \mathrm{keV}$. At low energies and high beam intensities, space charge effects are dominat and need to be included in beam dynamics studies. They cause significant emittance increase due to their large repulsive force. Thus we propose to use $\mathrm{H}_{2}{ }^{+}$ions as the design particle instead of protons. We will then use a stripper foil after extraction from the cyclotron to double the beam intensity by removing the binding electron. This idea reduces the required beam intensity to $5 \mathrm{~mA}$ of $\mathrm{H}_{2}^{+}$(10 mA of protons after stripping). 
Another advantage of employing an RFQ is the reduction of extraction voltage at the ion source. The role of the RFQ accelerator is for longitudinal bunching and acceleration, to accomplish a very compact system.

Table 1: Design results of the beam dynamics study of IsoDAR RFQ.

\begin{tabular}{|l|l|l|l|l|l|}
\hline Elements & Value & Unit & Elements & Value & Unit \\
\hline Frequency & 32.8 & $\mathrm{MHz}$ & Particle $(\mathrm{q} / \mathrm{A})$ & $\mathrm{H}_{2}^{+}$ & $1 / 2$ \\
\hline Beam energy & $15 \rightarrow 70$ & $\mathrm{keV}$ & Vane voltage & 20.14 & $\mathrm{kV}$ \\
\hline Length & 137.869 & $\mathrm{~cm}$ & No. of cells & 58 & \\
\hline Trans. rate & 97.270 & $\%$ & Oct. term & 0.070 & \\
\hline Inpt Tr. emit & 0.300 & $\pi . \mathrm{mm} . \mathrm{mrad}$ & Out Tr. emit & 0.343 & $\pi . \mathrm{mm} . \mathrm{mrad}$ \\
\hline min. aperture & 0.683 & $\mathrm{~cm}$ & Out Lo. emit & 40.24 & $\mathrm{keV}-\mathrm{deg}$ \\
\hline vanetip curv. & 0.930 & $\mathrm{~cm}$ & Peak power & 12.25 & $\mathrm{~kW}$ \\
\hline Duty rate & 20 & $\%$ & $\mathrm{E}_{\text {surface }}$ & $0.4^{*} \mathrm{E}_{\mathrm{k}}$ & $\mathrm{MV} / \mathrm{m}$ \\
\hline
\end{tabular}

The beam dynamics have been studied to satisfy the requirements using $50 \mathrm{k}$ particles in the PARMTEQM code [2]. Since modulation and synchronous phase in the low energy section are key parameters to determine the longitudinal emittance, adiabatic varied modulation and synchronous phase were applied to obtain high transmission rate and a small longitudinal emittance. In addition, smooth varied parameters were applied in the shaper and gentle buncher sections to avoid transverse emittance blow-up at the end of the bunching section due to a strong RF defocusing and octupole effects. Fig. 2 shows the beam dynamics results such as rms beam size and normalized rms transverse and longitudinal emittances along the beam axis. In order to obtain large acceptance at the entrance of the cyclotron, we have carefully chosen the cell parameters to be varied in smooth and small increments of modulation and synchronous phase in the beginning of the bunching section to minimize the longitudinal emittance.

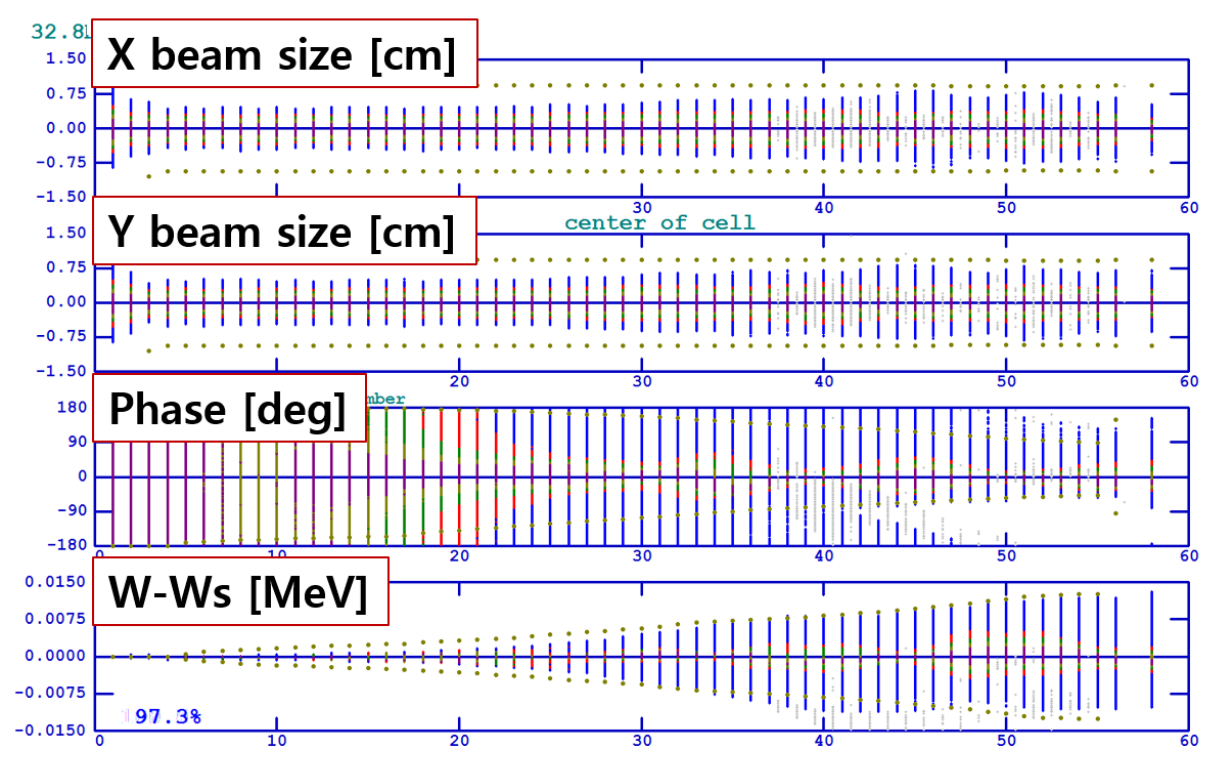

Figure 2 Beam dynamics results in the IsoDAR RFQ 
The resulting design parameters are presented in Fig. 3. The aperture $a$, vane-tip curvature $\rho$, and vane voltage $v$, are kept constant for easy manufacturing and field tuning. By optimizing the remaining design parameters, $97.27 \%$ of transmission rate has been obtained with a moderate length of $137.869 \mathrm{~cm}$. The estimated peak power consumption of the RFQ is based on a CST MWS simulation as $12.25 \mathrm{~kW}$ [3]. There will be a spiral inflector after the RFQ to bend the beam from the vertical direction onto the cyclotron median plane. During these $40 \mathrm{~cm}$ of travel, the particle distribution will grow in size in both transverse and longitudinal direction. Thus we propose to add a re-buncher cell after the RFQ acceleration section to focus the longitudinal beam distribution at the first accelerating gap of the cyclotron. The re-buncher is positioned in the exit transition cell of the RFQ. The study of the re-buncher in terms of beam dynamics and design is described in detail in the following section.
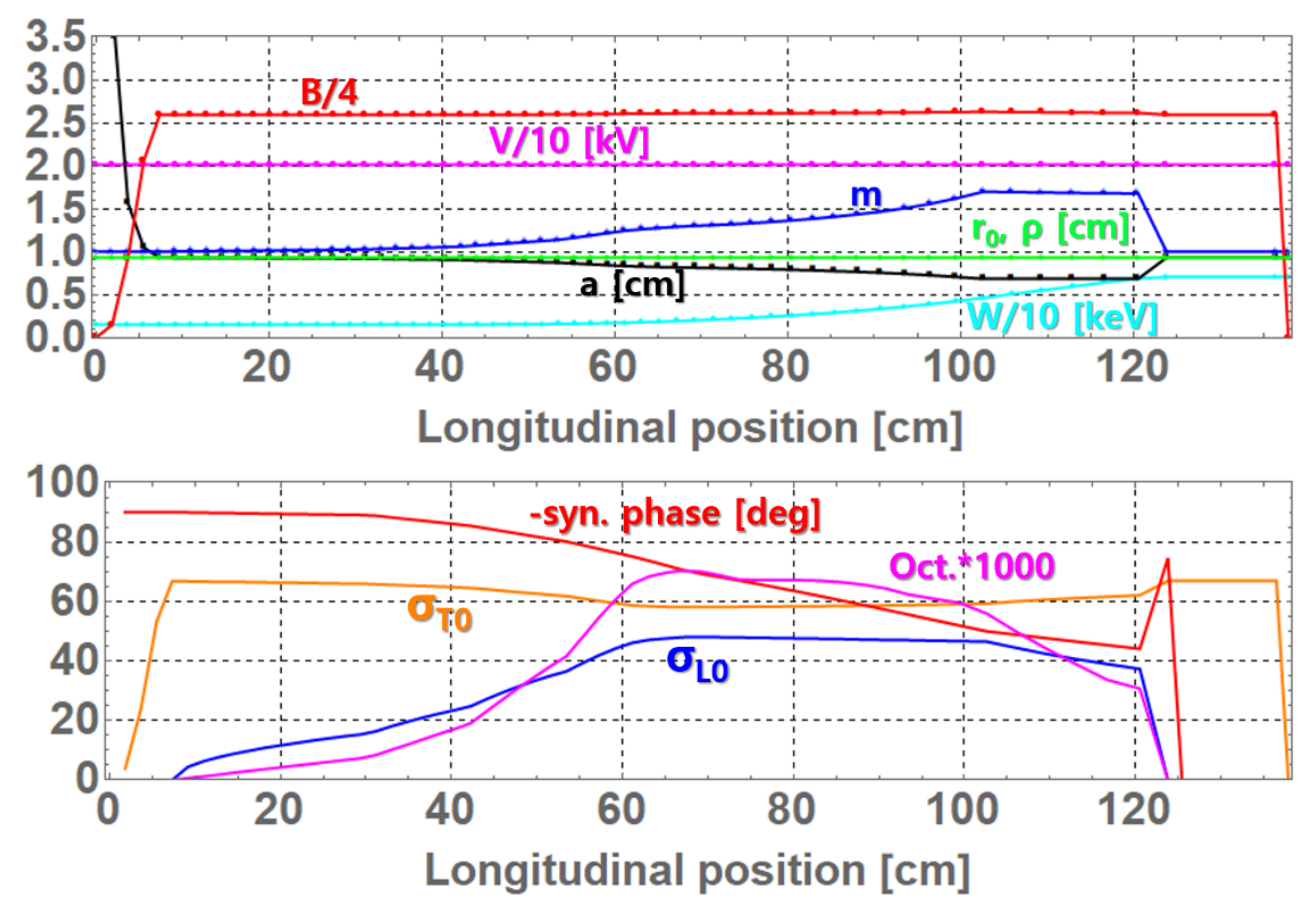

Figure 3 RFQ design parameters along the longitudinal position

\section{RFQ cavity design}

The operation frequency of the RFQ is determined by the cyclotron operating frequency (32.8 $\mathrm{MHz}$ ). Due to the low operation frequency and size requirements for embedding the cavity in the cyclotron yoke, the cavity design is chosen as a split-coaxial type. Since a split-coaxial RFQ cavity has a characteristic varied magnetic field strength along the beam axis, it has the advantage of the largest capacitance compared with other types of RFQ's and allows a small transverse diameter with low operation frequency [4]. The split-coaxial structure is composed such that two opposite vanes are connected to one of the end-plates and the other two vanes are connected to the other end-plate [5]. In addition to low frequencies at small diameters, this provides high stability of the electromagnetic field distribution, as the magnetic field encompasses all four vanes as a coaxial waveguide. The geometric cavity design parameters are shown in Figure 4 and the values are listed in Table 2. 


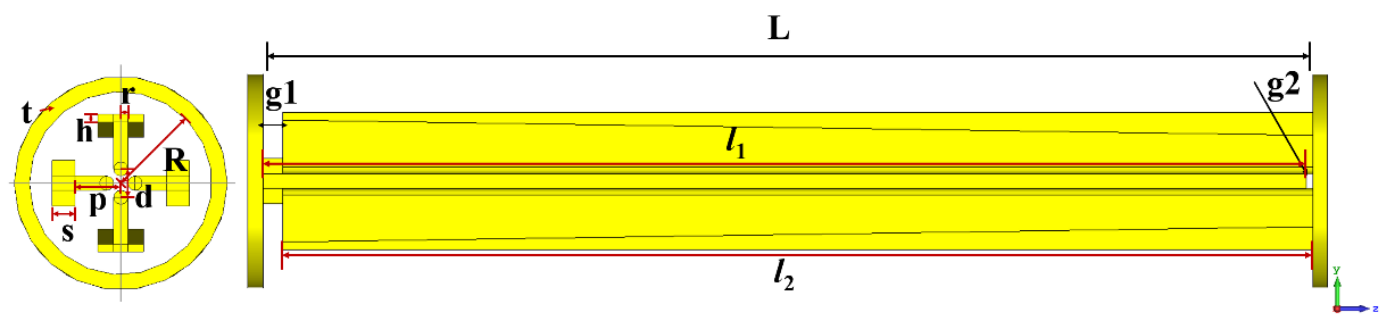

Figure 4: Geometry parameters for a split-coaxial type of RFQ cavity for IsoDAR.

Because the beam expands longitudinally between the RFQ exit and cyclotron central region, a re-buncher cell has been added to focus the beam in longitudinal direction. As there is not enough space to install a buncher and magnets after the RFQ cavity, the re-buncher is placed in the transition cell of the RFQ. In order to generate an efficient longitudinal electric field distributon, trapezoidal and sinusoidal buncher shapes were studied. Focal length and synchronoise phase for longitudinal bunching are optimized by modulation height and re-buncher position.

Table 2: The geometry parameters of the $R F Q$.

\begin{tabular}{|l|l|l|l|l|l|}
\hline Elements & Value & Unit & Elements & Value & Unit \\
\hline Cavity radius & 120.00 & $\mathrm{~mm}$ & Skirt max thickness & 30.00 & $\mathrm{~mm}$ \\
\hline Electrode radius & 9.30 & $\mathrm{~mm}$ & Skirt min thickness & 10.00 & $\mathrm{~mm}$ \\
\hline Vane skirt position & 60.00 & $\mathrm{~mm}$ & Electrode distance & 18.60 & $\mathrm{~mm}$ \\
\hline
\end{tabular}

Fig. 5 shows the two cases of electric field distribution of the trapezoidal and sinusoidal rebunchers. Due to the better bunching efficiency and shorter fringe field region, the trapezoidal rebuncher was chosen. The center position of the re-buncher cell is $1320 \mathrm{~mm}$ from the RFQ entrance and the peak electric field strength is $0.4 \mathrm{MV} / \mathrm{m}$ along the beam axis.

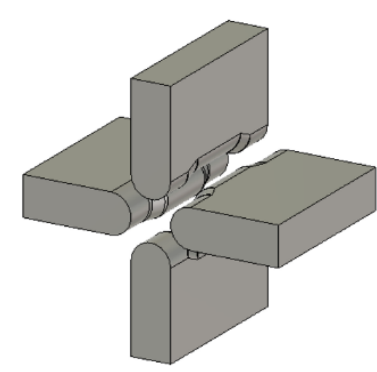

(a) Trapezoidal re-buncher

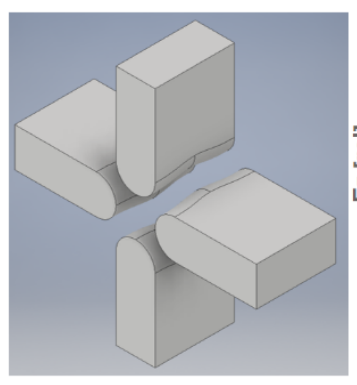

(b) Sinusoidal re-buncher

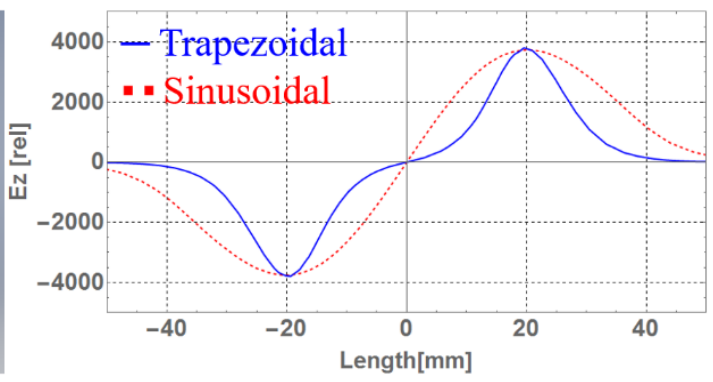

(c) Field distribution on axis

Figure 5 : The two investigated types of re-buncher cell shape (a, b) and field distribution (c).

\section{Conclusion}

As part of the IsoDAR injection system, an RFQ has been designed that satisfies the requirements of high bunching efficiency, compactness, high intensity beam transport, and matching into the cyclotron central region. Beam dynamics studies of the RFQ show high transmission rates of over $95 \%$ and a $40 \mathrm{keV}-\mathrm{deg}$ longitudinal emittance for a $6.5 \mathrm{~mA} \mathrm{H}_{2}{ }^{+}$beam. By adopting a split-coaxial type of RFQ, the compact transverse size necessary for embedding 
the RFQ in the cyclotron yoke is achieved. In order to reduce energy spread and beam size in longitudinal direction, a re-buncher cell is implemented in the transition cell of the IsoDAR RFQ. Fig. 6 shows the effect of the re-buncher cell by comparing the beam distribution with and without re-buncher cell (results obtained using the TRACK code [6]). Adding the re-buncher, the injection into the cyclotron using an RFQ could be further improved by a factor 3.7.

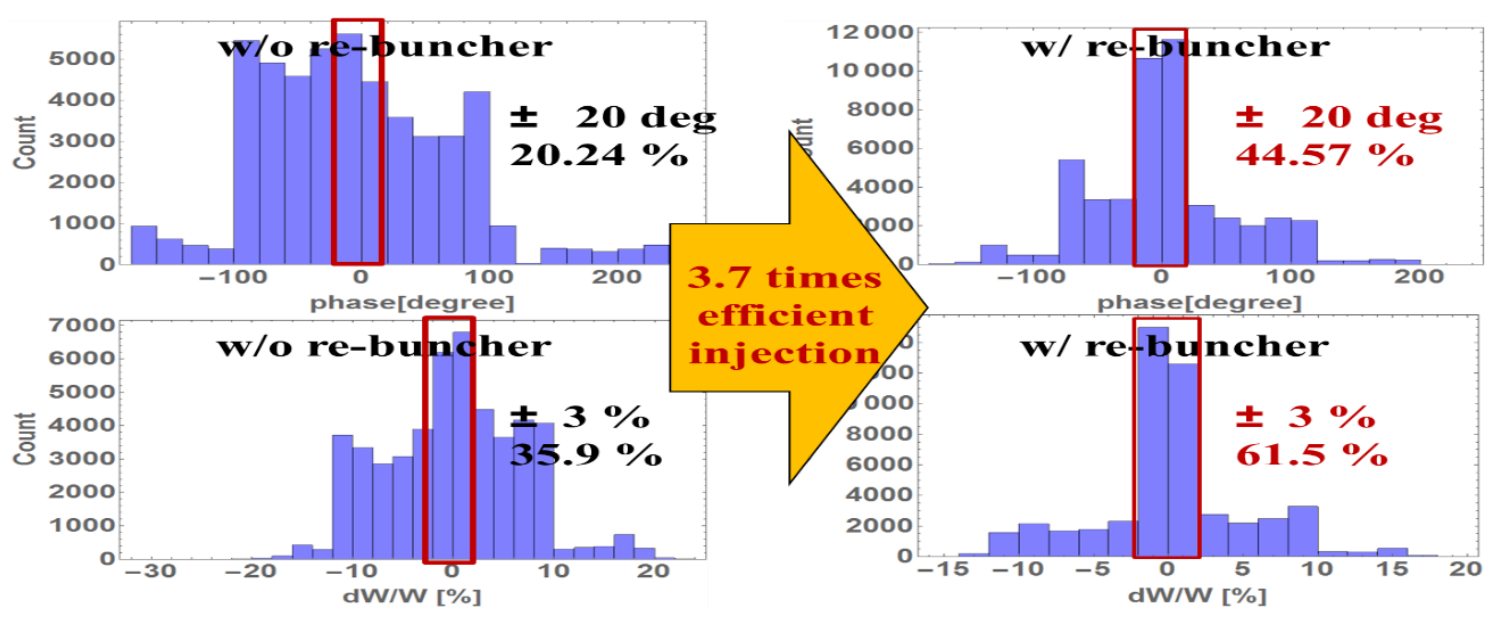

Figure 6 : Comparison result of re-buncher effects $n$ the partcle distributions

\section{Acknowledgements}

The RFQ-DIP project is supported by NSF grant \#PHY-1626069 and the Heising-Simons Foundation. Winklehner thanks the American Physical Societey and Indo-U.S. Science and Technology Forum for awarding him a travel grant through the Ph.D. student and Postdoc Visitation Program to go to Kolkata and collaborate on RFQ design with the RIB group at VECC.

\section{References}

[1] J. M. Conrad, M. H. Shaevitz, I. Shimizu, J. Spitz, M. Toups, L. Winslow, Precision -electron scattering measurements with IsoDAR to search for new physics, Phys. Rev. D89 (7) (2014) 072010. arXiv:1307.5081, doi:10.1103/PhysRevD.89.072010.

[2] K.R. Crandall, T. P. Wangler, PARMTEQ a beam dynamics code for the RFQ linear accelerator, in : Linear Accelerator and Beam Optics Codes, Vol. 177, AIP Publishing, 1988, pp 22-28.

[3] CST Microwave Studio. URL www.cst.com

[4] P. Ostroumov, K. Shepard, Design of 57.5 MHz CW RFQ structure for the rare isotope accelerator facily, Pramana-Journal of Physics 59 (6) (2002) 977-988.

[5] Y. Katayama, Bulletin of the Institute for Chemical Research, Kyoto University Vol. 61, No. 1 (1983)

[6] P. Ostroumov, K. Shepard, Correction of beam-steering effects in low-velocity superconducting quarter-wave cavities, Phys. Rev. ST. Accel. Beams 4 (2001) 110101. 